Fasting promotes HSC function

Prolonged fasting activates a metabolic switch to lipid- and ketone-based catabolism and decreases circulating insulin-like growth factor-1 (IGF-1), which has been shown to reduce chemotoxicity, although the reasons behind this effect are unclear. Myelosuppression is a side effect of chemotherapy, and Longo and colleagues have now discovered that the positive effects of prolonged fasting can partly be attributed to improved haematopoietic stem cell (HSC) function (Cell Stem Cell 6, 810-823; 2014).

The authors initially found that reduction of circulating IGF-1 levels in the blood was accompanied by a reduction in protein kinase A (PKA) signalling activity in several cell types associated with blood function. They observed that cycles of prolonged fasting followed by challenges with the chemotherapy agent cyclophosphamide reduced the mortality and apoptosis of long- and short-term HSCs as well as multipotent progenitors in the bone marrow. In addition, multi-lineage differentiation was improved in these animals compared with fed mice, in vitro and in transplantation experiments. This was also observed in cancer patients adopting prolonged fasting within a phase I clinical trial. These positive effects of prolonged fasting were independent of the chemotherapy treatment, as they were also present in aged animals, which naturally exhibit a reduction in HSC function and multilineage potential. The effects of prolonged fasting could be reproduced in mice lacking the growth hormone receptor, which also have low levels of IGF-1. Transplantation experiments showed that low levels of IFG-1 in animals led to a reduction in IGF-1-mediated PKA signalling, both in haematopoietic cells and in associated stromal cells. Strikingly, the researchers could restore haematopoietic function by reducing the levels of either IGF-R1 or the PKA catalytic subunit by siRNA. Conversely, the benefits were abolished if exogenous IGF-1 was added. It will be fascinating to see if the positive effects of fasting described here can be translated to a clinical setting. NLB

\section{Extra centrosomes provide route to invasion}

Centrosome amplification is associated with cancer, but it remains unclear how multiple

\title{
Mechanotransduction in collective cell migration
}

The Drosophila melanogaster ovary egg chambers contain nurse cells and an oocyte surrounded by epithelial follicle cells. During oogenesis, polar cells stimulate a group of epithelial cells, termed border cells, to move between nurse cells towards the oocyte. Successful border cell migration requires E-cadherin expression in both border and nurse cells. Montell and colleagues now provide an elegant characterization of the role of E-cadherin in this process (Cell 157, 1146-1159; 2014).

Using Drosophila strains with E-cadherin depleted in border, nurse or polar cells, they demonstrated that E-cadherin-based adhesions between border and nurse cells are necessary for directional migration of border cells, whereas E-cadherin depletion in polar cells disrupts border cell clusters. The authors generated transgenic flies expressing E-cadherin modified to include a Förster resonance energy transfer (FRET)-based tension sensor, to register mechanical force transduction across E-cadherin molecules. They observed a higher level of tension at the front of the border cell cluster, indicating higher adhesive strength and/ or actomyosin contractility at that site. Morphodynamic profiling of protrusion and retraction behaviour of border cells expressing dominant-negative forms of PVR (PDGF- and VEGF-receptor related) and EGFR (epidermal growth factor receptor) confirmed the known role of these receptors in guiding border cells. Comparing these profiles with those from expression of dominant-negative Rac and depletion of E-cadherin indicated that PVR and EGFR stimulate Rac activity and function upstream of E-cadherin in border cell guidance. Further tension sensor experiments revealed that the guidance receptors and Rac promote tension on E-cadherin at the front of the cluster. Experiments with a Rac FRET probe and Rac photoinhibition combined with depletion of adherens junction proteins showed that E-cadherin is involved in a positive feedback amplification of Rac signalling at the front of the border cell cluster to promote forward protrusion stabilization and to allow directional migration through E-cadherin-based adhesion between border cells.

centrosomes may affect tumorigenesis. Godinho, Pellman and colleagues have revealed that supernumerary centrosomes confer an invasive behaviour to epithelial cells in 3D culture, through a mechanism involving Rac1 activation (Nature 510, 167-171; 2014).

Overexpression of polo-like kinase 4 (Plk4) was used to induce centrosome amplification in the epithelial cell line MCF10A. The authors observed that this resulted in invasive protrusions in $3 \mathrm{D}$ culture and collective migration in an organotypic culture system. Centrosome amplification is known to lead to chromosome missegregation and aneuploidy, but as aneuploidy induced by other means did not result in invasion, the authors concluded that aneuploidy per se is not responsible for the phenotype. Instead they observed that cell-cell contacts were disrupted, cells were scattered, and the position and size of cell-cell junctions altered. Rac1 activation is known to cause similar phenotypes, and, using biochemical methods as well as a Racl biosensor, the authors found that centrosome amplification did indeed induce Rac1 activation. Importantly, Rac1 inhibition partly rescued the cell-cell adhesion effects and blocked the formation of invasive acini induced by centrosome amplification. Previous work had suggested that dynamic microtubules may influence Rac1 activation, and the authors found increased centrosomal $\gamma$-tubulin levels and microtubule nucleation associated with supernumerary centrosomes. Furthermore, depletion of CEP192, a centrosomal protein needed for interphase $\gamma$-tubulin recruitment, could prevent Rac1 activation and disrupt invasion induced by supernumerary centrosomes. Thus, in cells with extra centrosomes, increased centrosomal microtubule nucleation leads to Rac1 activation, disruption of cell-cell contacts, and invasive behaviour.

Although the authors note that centrosome amplification is likely to have other effects contributing to tumorigenesis, this study offers insight into how extra centrosomes can induce the invasive behaviour associated with aggressive tumours.

CKR 